Supporting Information

\title{
Magnetic stiffening in 3D cell culture matrices
}

Wen Chen, ${ }^{l}$ Ying Zhang, ${ }^{1}$ Jyoti Kumari, ${ }^{1}$ Hans Engelkamp ${ }^{1,2}$ and Paul H. J. Kouwer ${ }^{1, *}$

1. Radboud University, Institute for Molecules and Materials, Heyendaalseweg 135, 6525 AJ Nijmegen, The Netherlands. E-mail: p.kouwer@science.ru.nl

2. Radboud University, High Field Magnet Laboratory (HFML-EMFL), Toernooiveld 7, Nijmegen, The Netherlands 


\section{Materials preparation}

All chemicals were of analytical grade and used without further purification. Stock solutions were all prepared in deionized (18 $\mathrm{M} \Omega \mathrm{cm})$ and purified water (MilliQ) unless otherwise mentioned.

\section{Synthesis of small-size magnetic iron oxide nanospheres (SMIONSs)}

For the synthesis of the SMIONSs, a previously reported method was followed with minor modifications. ${ }^{1}$ In brief, a mixture of $\mathrm{FeCl}_{3} \cdot 6 \mathrm{H}_{2} \mathrm{O}(0.2 \mathrm{M}, 50 \mathrm{~mL})$ and $\mathrm{FeCl}_{2} \cdot 4 \mathrm{H}_{2} \mathrm{O}(0.1 \mathrm{M}, 50$ $\mathrm{mL})$ was prepared under vigorous stirring. $1 \mathrm{M} \mathrm{NaOH}$ solution $(40 \mathrm{~mL})$ was added dropwise. The solution was heated at $90{ }^{\circ} \mathrm{C}$ for $4 \mathrm{~h}$. Note that the deionized and purified water (MilliQ) that was used in the experimental preparation was deoxygenated by bubbling through Ar gas for $1 \mathrm{~h}$. The SMIONSs were separated by placing a magnet under reaction flask and washing with water and ethanol for 3 times, respectively. The washed SMIONSs were functionalized by poly(acrylic acid sodium salt) (PAASS, $2 \mathrm{mg} \mathrm{mL}^{-1}, 50 \mathrm{~mL}$ ) overnight at room temperature, followed by 3-fold water and ethanol washings. Then the collected SMIONSs were dried under vacuum overnight for further use. Before any use, the iron oxide nanomaterials were sonicated for 10 mins at room temperature.

\section{Synthesis of large-size magnetic iron oxide nanospheres (LMIONSs)}

For the synthesis of the LMIONSs, the protocol modified by Yong et al. was used. ${ }^{2}$ In short, $\mathrm{FeCl}_{3} \cdot 6 \mathrm{H}_{2} \mathrm{O}(1.8 \mathrm{~g})$ and $\mathrm{NaOAc}(2.04 \mathrm{~g})$ were dissolved in ethylene glycol $(70 \mathrm{~mL})$ with vigorous magnetic stirring for 30 mins. The solution was transferred into a Teflon-lined stainless-steel autoclave and heated at $200{ }^{\circ} \mathrm{C}$ for 12 hours. The purification and functionalization procedure were the same as what was described for SMIONSs. The collected LMIONSs were dried under vacuum overnight for further use.

\section{Synthesis of magnetic iron oxide nanorods (MIONRs)}

For the synthesis of the MIONRs, an established simple two-step method ${ }^{3}$ was slightly modified. First, FeOOH nanorods were synthesized by heating an aqueous solution of $\mathrm{FeCl}_{3} \cdot 6 \mathrm{H}_{2} \mathrm{O}(0.1 \mathrm{M}$, $70 \mathrm{~mL})$ and $\mathrm{HCl}(1 \mathrm{M}, 4.8 \mathrm{~mL})$ into a Teflon-lined stainless-steel autoclave $(100 \mathrm{~mL})$ at $87{ }^{\circ} \mathrm{C}$ for 24 hours. The solution was centrifugated and washed with water for 3 times. The purified $\mathrm{FeOOH}$ nanorods were functionalized by PAASS overnight. Second, the above aqueous dispersion was injected into a pre-heated $\left(200{ }^{\circ} \mathrm{C}\right)$ diethylene glycol $(70 \mathrm{~mL})$ solution containing PAASS as surface protectant and transferred into Teflon autoclave for hydrothermal treatment at $220{ }^{\circ} \mathrm{C}$ for $8 \mathrm{~h}$. The product was separated from the solution by a magnet and purified by washing with water and ethanol for 3 times, respectively. Then the collected $\mathrm{Fe}_{3} \mathrm{O}_{4}$ nanorods were dried under vacuum overnight for further use. 


\section{Synthesis of PIC and azide-PIC and gel preparation}

Polyisocyanides (PIC) were synthesized using a previously reported procedure. ${ }^{4}$ The polymerization of the tri(ethylene glycol)-grafted isocyano-( $(D)$-alanyl-( $L$ )-alanine monomer 1 was catalyzed by nickel perchlorate (total monomer:catalyst ratio for all polymers was 1000:1). For the cell culture, the PIC polymers were functionalized with cell-adhesive peptides. ${ }^{5}$ Functionalized polymers were obtained by co-polymerization of the tri(ethylene glycol)-grafted isocyano- $(D)$ alanyl-( $L$ )-alanine monomer $\mathbf{1}$ and the azide-appended monomer $\mathbf{2}$ (ratio 19:1), catalyzed by nickel perchlorate (total monomer:catalyst ratio for all polymers was 1000:1). Details of the preparation, purification, peptide preparation ${ }^{6}$ and subsequent functionalization ${ }^{7}$ have been published before.

For mechanical measurements, a stock solution of PIC polymer was dissolved in MilliQ at $4{ }^{\circ} \mathrm{C}$ overnight. Samples were prepared from the stock solution by diluting with MilliQ to the desired concentration.

For the cell culture, the desired amount of sterile HBSS (Sigma Aldrich, Cat. \#H4891, prepared by dissolving Hank's Balanced Salts $\left(1 \mathrm{~g}\right.$ in $1 \mathrm{~L}$ sterile $\mathrm{H}_{2} \mathrm{O}$ ) was added to the solid, sterilized (UV, $5 \mathrm{~min}$ ) polymer. After overnight soaking at $4{ }^{\circ} \mathrm{C}$, the mixture was shaken vigorously for a few seconds and a transparent solution was formed. During the whole study, a single large batch of PIC polymer solution was prepared, aliquoted and frozen at $-20{ }^{\circ} \mathrm{C}$ until use. Before each experiment, the frozen gels were firstly placed on ice to thaw.

\section{Synthesis of polyacrylamide gel}

For the synthesis of the polyacrylamide (PAAM) gel, a previously reported method was followed. ${ }^{8}$ Stock solutions of acrylamide (AAM, 3.0 M, $1 \mathrm{~mL}$ ), $N, N^{\prime}$-methylenebis(acrylamide) (MBAA, 0.1 $\mathrm{M}, 1.144 \mathrm{~mL}$ ) and potassium peroxodisulfate (PS, $80 \mathrm{mM}, 3.107 \mathrm{~mL}$ ) were prepared in MilliQ water. To obtain a PAAM gel with a similar storage modulus as the PIC hydrogels, the molar ratio of AAM $(84.5 \mu \mathrm{L})$ to MBAA $(83.4 \mu \mathrm{L})$ was set to $100: 3$. To the above mixture solution, $250 \mu \mathrm{L}$ of PS, $1 \mu \mathrm{L}$ of tetramethylethylenediamine (TEMED), and $182.2 \mu \mathrm{L}$ of MilliQ were added to initiate the polymerization. The solution was transferred onto the rheometer at $25^{\circ} \mathrm{C}$ within $2 \mathrm{~min}$, followed by rheology measurement at $25^{\circ} \mathrm{C}$.

\section{Magnetic hydrogels preparation}

The $\mathrm{PIC} / \mathrm{Fe}_{3} \mathrm{O}_{4}$ nanoparticle (NP) hybrid hydrogels (SMIONSs, LMIONSs and MIONRs gels) were prepared by mixing a cold $\left(4^{\circ} \mathrm{C}\right)$ PIC stock solution with a cold stock solution of magnetic nanomaterials, respectively. To prepare the hybrid hydrogels with different concentrations of PIC or $\mathrm{Fe}_{3} \mathrm{O}_{4} \mathrm{NPs}$, the desired amount of MilliQ was added to dilute the mixture to $0.2 \mathrm{~mL}$. The solutions were mixed homogeneously and placed on the precooled rheometer $\left(5^{\circ} \mathrm{C}\right)$ for mechanical analysis. The PAAM/MIONPs hydrogels were prepared by adding the $\mathrm{Fe}_{3} \mathrm{O}_{4} \mathrm{NPs}$ to the AAM polymerization solution at room temperature, transferring the mixture to rheometer $25^{\circ} \mathrm{C}$ and incubating for $1 \mathrm{~h}$ to complete the polymerization and gel formation. 


\section{Rheology and magneto-rheology}

The mechanical properties were analyzed on stress-controlled rheometer (TA Instruments, Discovery HR-2) in a $20 \mathrm{~mm}$ parallel plate geometry (gap $500 \mu \mathrm{m})$. For the magneto-rheological studies, the TA temperature-controlled magneto-rheology accessory was used in a steel parallel plate geometry with a plate with diameter of $20 \mathrm{~mm}$ and a gap of $500 \mu \mathrm{m}$. Cold PIC or composite solutions were loaded onto the rheometer at $5{ }^{\circ} \mathrm{C}$ and heated to $37{ }^{\circ} \mathrm{C}$. After equilibration $(10$ minutes) an external magnetic field was ramped up from $0 \mathrm{~T}$ to $1 \mathrm{~T}$ at the desired rate (often in 5 mins), while measuring the mechanical properties in the linear viscoelastic regime (strain $\gamma=2 \%$, frequency $\omega=1 \mathrm{~Hz}$ ). For reversibility studies, the PIC/MIONPs gels were repeatedly subjected to magnetic fields between $0 \mathrm{~T}$ and $1 \mathrm{~T}$. All rheology data shown in this work is recorded after the gel was formed at $37^{\circ} \mathrm{C}$ unless mentioned otherwise.

\section{Transmission Electron Microscopy (TEM)}

For TEM analysis of SMIONSs, LMIONSs and MIONRs, a JEOL JEM-1400 operating at $120 \mathrm{kV}$ was used. The samples were suspended in an ethanol solution and mechanically mixed (Ultrasonic agitation for 10 mins), after which they were dropped on a $\mathrm{Cu}$ TEM grid and air dried. The size distributions of MIONPs were calculated by ImageJ software.

\section{Cryo-Scanning Electron Microscopy (cryo-SEM)}

For SEM analysis of PIC/MIONRs gels, JEOL 6330 Cryo Field Emission operating at $12 \mu \mathrm{A}$ was used. The hybrid gel samples were first fixed with nitrogen slush and transferred to the cryochamber. To observe the inner structure, the frozen materials were cleaved, sublimated and coated with gold-palladium inside the vacuum cryo chamber.

\section{Particle retention}

For quantification of MIONR retention in the composite, a gel with a high concentration of MIONRs (25 mg mL $\mathrm{mL}^{-1}$ ) was prepared, similar to the materials used for cell culture $(30 \mu \mathrm{L}$ $\mathrm{PIC} /$ Matrigel/MIONRs with or without magnetic field). The samples were washed with MilliQ water for more than three time (1 time per hour) and subsequently ionized for elemental analysis via nitric acid treatment. The ICP-atom emission spectrometer Plasma was used for the iron

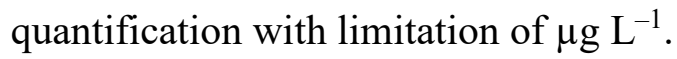

\section{Live-dead experiments}

Human foreskin fibroblasts cells were cultured in DMEM containing 10\% of FBS and 1\% penicillin-streptomycin. The cytotoxic effects of MIONRs on the cells were analyzed through a live-dead staining protocol using Calcein-AM (Invitrogen, LOT1933362) and TOTO ${ }^{\mathrm{TM}_{-}} 3$ (Invitrogen, LOT1985289). Briefly, human foreskin fibroblasts cells, $6 \times 10^{4}$ cells $\mathrm{mL}^{-1}$ were seeded on an Ibidi micro plate in presence of different concentration of MIONRs $\left(0 \mathrm{mg} \mathrm{mL}^{-1}, 3\right.$ $\mathrm{mg} \mathrm{mL}^{-1}, 25 \mathrm{mg} \mathrm{mL}^{-1}$ and $50 \mathrm{mg} \mathrm{mL}^{-1}$. At day 3, cells were incubated with Calcein-AM (2mM, 
1:1000 dilution) and TOTO ${ }^{\mathrm{TM}}-3\left(1 \mathrm{Mm}, 1: 1000\right.$ dilution) for $1 \mathrm{~h}$ at $37^{\circ} \mathrm{C}$ in a $5 \% \mathrm{CO}_{2}$ incubator. Cells were then washed with PBS and analyzed under confocal microscopy.

\section{MCF10A Cell culture}

MCF 10A cells were used as cell line to study the magneto-mechanical response of the matrix. All matrices in this study were composed of PIC-RGD $\left(2 \mathrm{mg} \mathrm{mL}^{-1}\right)$ and Matrigel $\left(3.52 \mathrm{mg} \mathrm{mL}^{-1}\right)$ and with or without added MIONRs $\left(25 \mathrm{mg} \mathrm{mL}^{-1}\right)$ added. Matrigel was added to a cold PIC-RGD solution, and mixed about 30 times with a pipette without generating bubbles. Then a welldispersed cold MIONRs solution was added and the mixture was mixed again, keeping the matrix cold on ice. Freshly MCF $10 \mathrm{~A}$ cells $(10,000-30,000$ cells per $50 \mu \mathrm{L}$ gel) were mixed with the above solutions and seeded at the bottom of 8-well plates. After gels had formed a disk-shaped gel in the incubator at $37^{\circ} \mathrm{C}$ for $1 \mathrm{~h}, 700 \mu \mathrm{L}$ culture medium was added per well and the constructs were cultured in a $37{ }^{\circ} \mathrm{C}$ humidified atmosphere under $5 \% \mathrm{CO}_{2}$. To apply a magnetic field during the cell culture, the well plate was placed on top of a neodymium permanent disk magnet during the entire cell experiment (approximate $450 \mathrm{mT}$ of field strength at the well plate), as shown in Figure S14. For the control cell experiment, the 8 -well plates were in the absence of an external magnetic field. The culture medium was refreshed every three days.

\section{Immunofluorescence analysis}

For immunofluorescence analysis, MCF 10A cell clusters generated within PIC-RGD hybrid hydrogels were fixed with 4\% paraformaldehyde (PFA) in PBS for $1 \mathrm{~h}$ at room temperature. PBS was removed by centrifuging and the pellet was incubated in $1.5 \%$ eosin for 5 min at room temperature. Following washing with PBS, the pellet was resuspended in $2.25 \%$ agar solution around $80-90{ }^{\circ} \mathrm{C}$. The hot agar solutions with cell clusters were centrifuged at $7200 \mathrm{rpm}$ for $2 \mathrm{~min}$. The solidified agar solutions with cell clusters were embedded in paraffin. Sections of $2 \mu \mathrm{m}$ thickness were cut using a microtome. Individual sections were mounted onto superfrost slides and dried overnight at $37^{\circ} \mathrm{C}$. After deparaffination with Histochoice (VWR, Cat. \#H103-4L) twice for $10 \mathrm{~min}$ each, samples were rehydrated with $100 \%(2 \times 1 \mathrm{~min}), 96 \%(2 \times 1 \mathrm{~min})$, and $70 \%(1 \times 1$ $\mathrm{min})$ ethanol, followed by washes with tap water $(2 \times 1 \mathrm{~min})$. The slides were then heated for 15 min in citrate buffer ( $\mathrm{pH}$ 6.0) (Dako, Cat. \#S1699) in a microwave oven for antigen retrieval. After cooling down for $1 \mathrm{~h}$, the samples were then blocked in 2\% BSA/PBS at room temperature for 1 $\mathrm{h}$. Primary antibody incubation was performed in $1 \% \mathrm{BSA} / \mathrm{PBS}$ at room temperature for $2 \mathrm{~h}$. The following primary antibodies were used: rabbit E-cadherin (Cell Signaling Technology, Cat. \#3195, 1:200). Secondary antibody incubation was in 1\% BSA/PBS at room temperature for $1 \mathrm{~h}$, followed by three PBS washes. Alexa 488-conjugated secondary antibodies (Invitrogen, Cat. \#A21206, 1:400) were used. All immunofluorescence experiments were performed with negative controls where relevant isotype was added (Rabbit isotype: Cell Signaling Technology, Cat. \#3900, 1:15000). The samples were then incubated with DAPI $\left(5 \mu \mathrm{g} \mathrm{mL}{ }^{-1}\right)$ at room temperature for $10 \mathrm{~min}$, followed by three PBS washes. The slides were mounted in anti-fade medium 
(Fluoromount $\mathrm{W}$ for microscopy, Serva), and images were acquired using a Leica DM6000 microscope (Leica). Acquired images were processed by Fiji.

\section{Quantification of morphology change}

For the quantification of the morphology change, we used three parameters (aspect ratio, roundness and solidity) to compare the four conditions (with or without MIONRs, with or without magnetic field). For each group, we analyzed 6-15 cell clusters. The aspect ratio is the ratio of length to height of an ellipse fitted to the cellular structure. The roundness is to measure how closely the shape of the spheroid approaches that of a circle. The solidity evaluates the smoothness of the spheroids' surface. The analysis is performed by ImageJ.

\section{Dynamic kinetics under external magnetic field}

Transient magnetorheological dynamics of gels is seldomly studied but gives information on the mechanism of the process. Figure S10 illustrates the dynamic evolution of storage modulus with stepwise change in magnetic field. The results fit well to a bi-exponential equation: ${ }^{9}$

$$
G^{\prime}(t)=G_{0 \mathrm{~T}}^{\prime}+\Delta G_{\max }^{\prime}\left(A_{1} e^{-t / \tau_{1}}+A_{2} e^{-t / \tau_{2}}\right)
$$

where $G^{\prime}(t)$ is the time-dependent storage modulus, $G_{0 \mathrm{~T}}^{\prime}$ is the gel's storage modulus in the absence of the field, $\Delta G_{\max }^{\prime}$ is the equilibrium storage modulus at a magnetic flux density of $1 \mathrm{~T}, t_{1}$ and $t_{2}$ are characteristic time scales and $A_{1}$ and $A_{2}$ are pre-factors. The fastest process of the two terms is associated to the formation of nanoparticle chains, whereas the slower process is attributed to cluster formation of the nanoparticles in dispersions. ${ }^{9}$ All experimental data was fitted in a single step where $t_{1}$ was chosen equal for all data sets. The fits are shown in Figure S10 and fitting parameters are given in Tables S2 and S3.

Variation of the MIONR concentration. The fitting procedure yields $t_{1}=6.6 \mathrm{~s}$. The secondary interaction process is slower. The decrease of $t_{2}$ with increasing $C_{\text {MIONR }}$ is consistent with the idea that this interaction becomes easier at higher concentrations of MIONRs. ${ }^{9}$

Variation of the PIC concentration. The fitting results show that the secondary interaction slows down for increasing PIC concentrations with $t_{2}$ increasing 5-fold between $C_{\text {PIC }}=0.25-3 \mathrm{mg} \mathrm{mL}^{-}$

1 . The result indicates that this interaction is hampered by the increasingly dense polymer network. Note that the data at $C_{\text {PIC }}=4 \mathrm{mg} \mathrm{mL}^{-1}$ was not fitted since the contribution of magnetic stiffening in this sample was very small.

\section{Internal force calculation}

The internal stress inside the magnetic-stressed hybrid was calibrated against the moduli of the externally stressed PIC gels (Figure 3A, dash lines) that similarly induces stiffening. It allows us to determine the average generated internal stress $\sigma_{\text {int }}$. Note that in this approach, the local differences in the entire network are averaged over the whole sample to give a consistent macroscopic response. 
We then use the approximated internal stress $\sigma_{\text {int }}$ generated by the magnetic field on MIONRs to quantify the average force $F_{\text {int }}$ on each PIC bundle, again averaged over the entire sample: ${ }^{10}$

$$
F_{\text {int }}=\frac{\sigma_{\text {int }}}{\rho_{\text {PIC }}}
$$

where $\rho_{\text {PIC }}$ is the PIC bundle density in length per volume, which is defined as:

$$
\rho_{\text {PIC }}=\frac{N_{\mathrm{A}} l_{\mathrm{M}}}{M} \frac{C_{\mathrm{PIC}}}{N}
$$

in which $N_{\mathrm{A}}$ is Avogadro's number, $l_{\mathrm{M}}$ is the length per monomer unit projected along the polymer backbone $\left(l_{\mathrm{M}}=0.25 \mathrm{~nm}\right), M$ is the molecular weight of isocyanide monomer $\left(M=0.316 \mathrm{~kg} \mathrm{~mol}^{-}\right.$ $\left.{ }^{1}\right), C_{\text {PIC }}$ is the PIC concentration in the hybrid (in $\mathrm{kg} \mathrm{m}^{-3}$ ) and $N$ is the average number of polymers chains per polymer bundle ( $N=7.3$, determined by SAXS measurements $\left.{ }^{4}\right)$.

We note that forces in reconstituted crosslinked F-actin networks induced by actomyosin contraction, ${ }^{11}$ were measured in an analogous way, which gave rise to values of about $F_{\text {int }} \approx 1 \mathrm{pN}$, quite similar to the magnetic forces in the PIC gels.

\section{Magnetic force calculation}

To determine if the MIONRs in a magnetic field are able to generate enough force to realize matrix stiffening, we theoretically estimated the maximum magnetic force. In the presence of an external magnetic field, two components of magnetic force on a ferromagnetic nanorod (NR) are generated. One is a linear force induced by the field gradient, and the other is magnetic alignment force caused by magnetic torque.

\section{The linear magnetic force}

The linear magnetic force $F_{\text {mag }}$ exerted per NR is primarily given by the field gradient (Figures S12 and S18): ${ }^{12}$

$$
F_{\mathrm{mag}}=\mu_{\mathrm{NR}} \cdot L(B) \cdot \frac{\mathrm{d} B}{\mathrm{~d} h}
$$

Here, $\mathrm{d} B / \mathrm{d} h$ is the slope of the magnetic field strength $B$ as a function of distance $h$ to the center of the magnet, $\mu_{\mathrm{NR}}$ is the total magnetic moment of one magnetic NR, which is given by:

$$
\mu_{\mathrm{NR}}=V \cdot m_{\mathrm{s}}
$$

where $V$ is the volume of an average nanorod, $m_{\mathrm{s}}$ is the spin magnetic moment, which is given by:

$$
m_{\mathrm{s}}=M_{\mathrm{s}} \cdot \rho_{\mathrm{Fe}_{3} \mathrm{O}_{4}}
$$

where $M_{\mathrm{s}}$ is the saturation magnetization, ${ }^{3} \rho_{\mathrm{Fe}_{3} \mathrm{O}_{4}}$ is the density of the nanorods, which is calculated by Archimedes' principle. The term $L(B)$ is the average degree of alignment of the particle in the field, which is given by Langevin function: 


$$
L(B)=\operatorname{coth}\left(\frac{\mu_{\mathrm{NR}} B}{k_{\mathrm{B}} T}\right)-\frac{k_{\mathrm{B}} T}{\mu_{\mathrm{NR}} B}
$$

where $k_{\mathrm{B}}$ is the Boltzmann constant and $T$ is the absolute temperature. For the NRs in this work, $L(B)$ is very close to unity. Based on the above calculations, the linear force amounts to values around $F_{\text {mag }}=10^{-14} \mathrm{~N}$.

\section{The magnetic alignment force}

In addition, we calculate the maximum torque based on the assumption that the nanorods rotate parallel to the magnetic field direction. The magnetic moment is defined as a vector relating the aligning torque per NR from an external magnetic field to the field vector itself, which is given by the equation:

$$
\tau=\mu_{\mathrm{np}} \times B=\left|\mu_{\mathrm{NR}}\right| \cdot|B| \cdot \sin \theta
$$

where $\theta$ is the misalignment angle. The maximum torque, therefore is given by $\tau_{\max }=\mu_{\mathrm{np}} B$, which amounts to values around $10^{-16} \mathrm{~N} \mathrm{~m}$. The maximum magnetic alignment force can be calculated by:

$$
F_{M}=\frac{\tau_{\mathrm{max}}}{L_{\mathrm{NR}}}
$$

where $L_{\mathrm{NR}}$ is the length of the NRs. The maximum alignment force therefore amounts to values around $F_{\mathrm{M}}=2 \cdot 10^{-10} \mathrm{~N}$. As the magnetic stiffening in LMIONSs and MIONRs are almost the same (Figure 1D), the magnetic stiffening in the composites is largely independent on the shape of nanoparticles, which means that the torque is expected to have a minor contribution to the magnetic stiffening effects.

\section{Estimation of the maximum magnetic force inside the network}

In order for the network to deform and stiffen, a minimum force is required. To determine if the magnetic response of the nanoparticles is large enough, we estimated the total maximum magnetic force in the sample, assuming that we can sum the contribution of all particles. Then the total magnetic force exerted on an average PIC fiber amounts to:

$$
F_{\mathrm{m}}=n_{\mathrm{NRs}} \cdot F_{\mathrm{mag}}
$$

where $n_{\mathrm{NRs}}$ is the number of nanorods per PIC fiber, which is defined as:

$$
n_{\mathrm{NRs}}=\frac{N_{\mathrm{NRs}}}{N_{\text {fibers }}}
$$

with $N_{\mathrm{NRs}}$ and $N_{\text {fibers }}$ being the total number of nanorods and PIC fibers, respectively. The last two quantities are defined by: 


$$
N_{\mathrm{NRs}}=\frac{N_{\mathrm{A}} C_{\mathrm{NRs}} V}{M_{\mathrm{NRs}}}
$$

where $C_{\mathrm{NRs}}$ is the concentration of nanorods and $V$ is the volume of the mixture solution, $M_{\mathrm{NRs}}$ is the molecular weight of nanorods; and

$$
N_{\text {fibers }}=\frac{N_{\mathrm{A}}}{M_{\mathrm{PIC}}} \frac{C_{\mathrm{PIC}} V}{N} .
$$
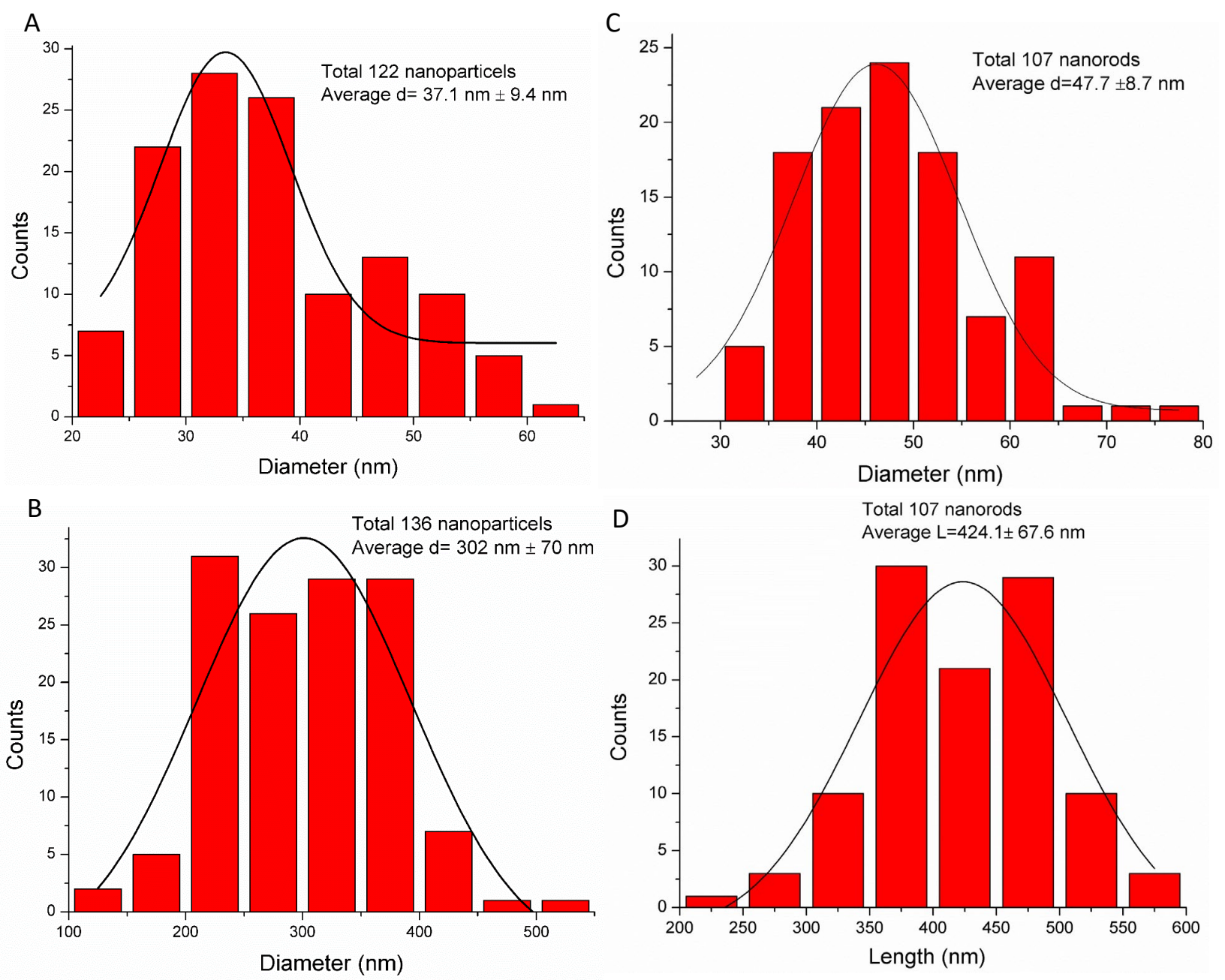

Figure S1. Size distribution of nanoparticles. A) Diameter distribution of SMIONSs. B) Diameter distribution of LMIONSs. C) and D) The diameter and length distributions of MIONRs, respectively. 

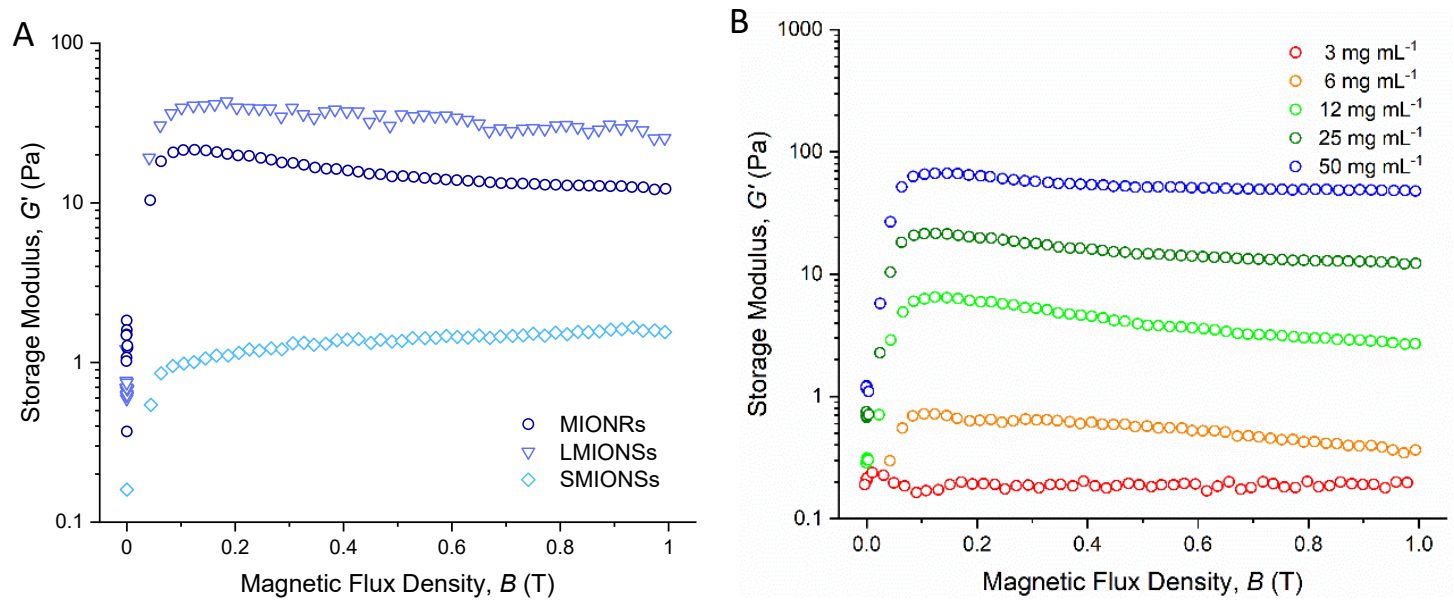

Figure S2. (A) Storage modulus $G^{\prime}$ of ferrofluids (no PIC) of MIONRs, LMIONSs and SMIONSs $\left(25 \mathrm{mg} \mathrm{mL}^{-1}\right.$ in MilliQ, $T=25^{\circ} \mathrm{C}$ ) as a function of flux density $B=0-1 \mathrm{~T}$. (B) Storage modulus $G^{\prime}$ of MIONR ferrofluids at different concentrations at flux density $B=0-1 \mathrm{~T}$.

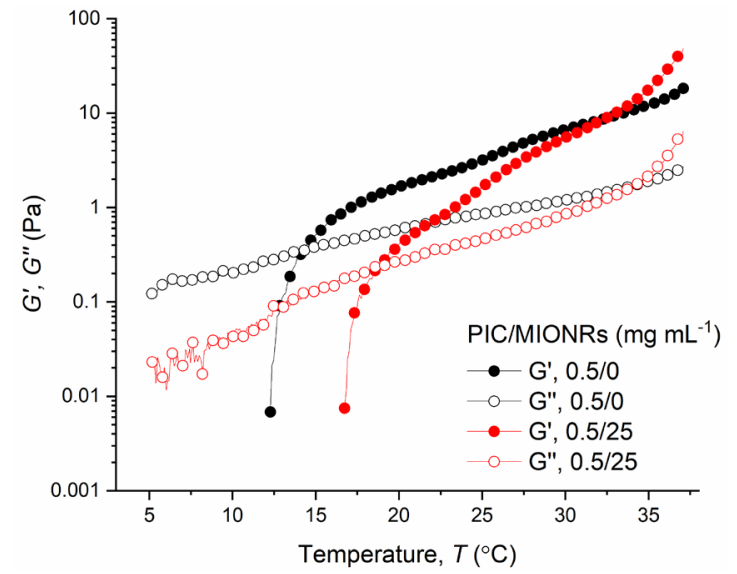

Figure S3. The storage modulus $G^{\prime}$ (solid signals) and loss modulus $G^{\prime \prime}$ (hollow signals) as a function of temperature. The thermally induced gelation process commences around $15-18{ }^{\circ} \mathrm{C}$. The incorporation of MIONRs only slightly contributed to the stiffness of the gel network. 


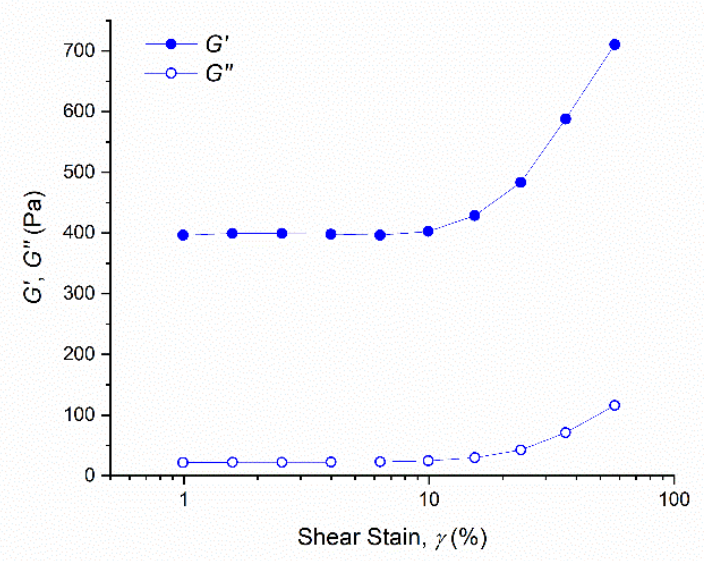

Figure S4. The storage modulus $G^{\prime}$ and loss modulus $G^{\prime \prime}$ of a PIC hydrogel $\left(2 \mathrm{mg} \mathrm{mL}^{-1}, T=37^{\circ} \mathrm{C}\right)$ as a function of shear strain.
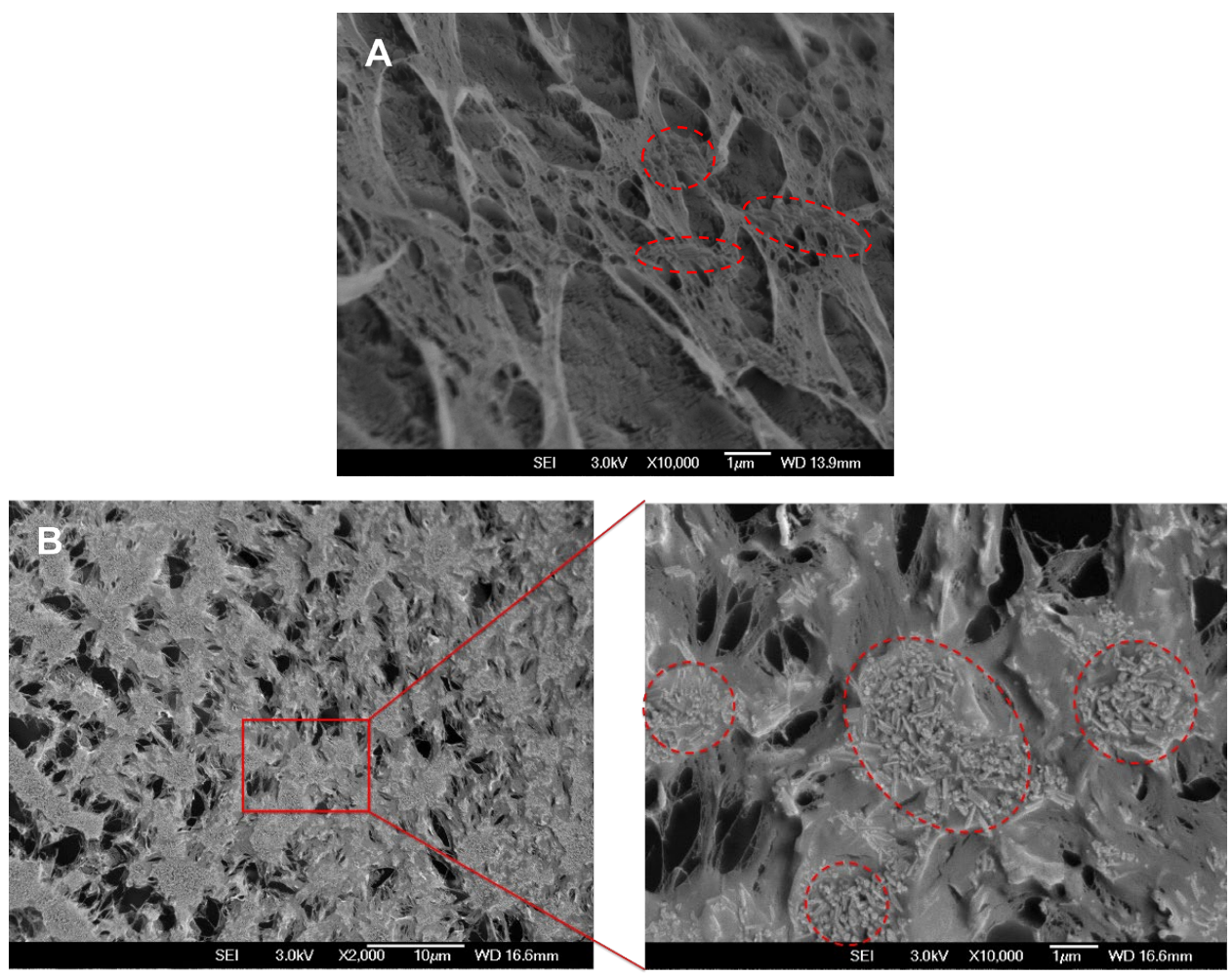

Figure S5. Cryo-SEM images of a PIC/MIONRs composite before (A) or after removing an external magnetic field (B). The scale bar is $1 \mu \mathrm{m}$ in Figure A. The scale bar in Figure B is $10 \mu \mathrm{m}$ (left) and $1 \mu \mathrm{m}$ (right). In the regions marked with the red dash lines, the MIONRs were randomly dispersed in the gel network. After removing magnetic fields, the MIONRs are still located inside the network. Conditions: nanorod concentration $25 \mathrm{mg} \mathrm{mL}^{-1}$, PIC concentration $0.5 \mathrm{mg} \mathrm{mL}^{-1}$. 
A
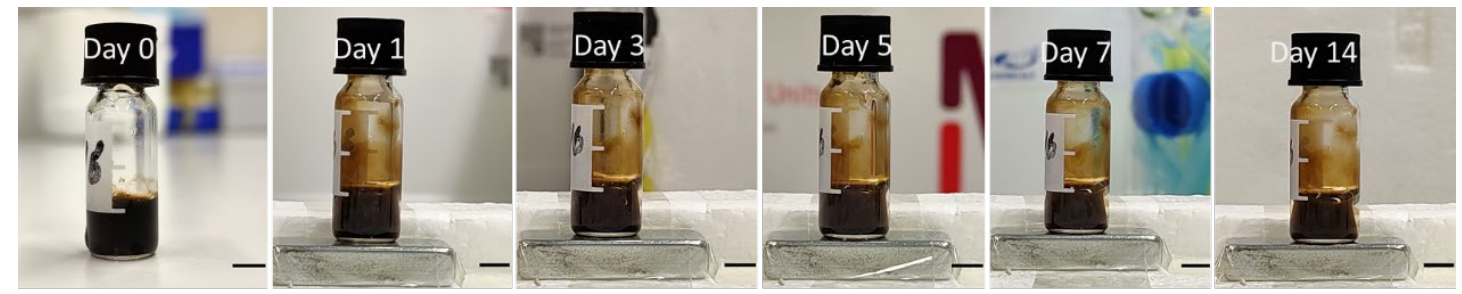

B

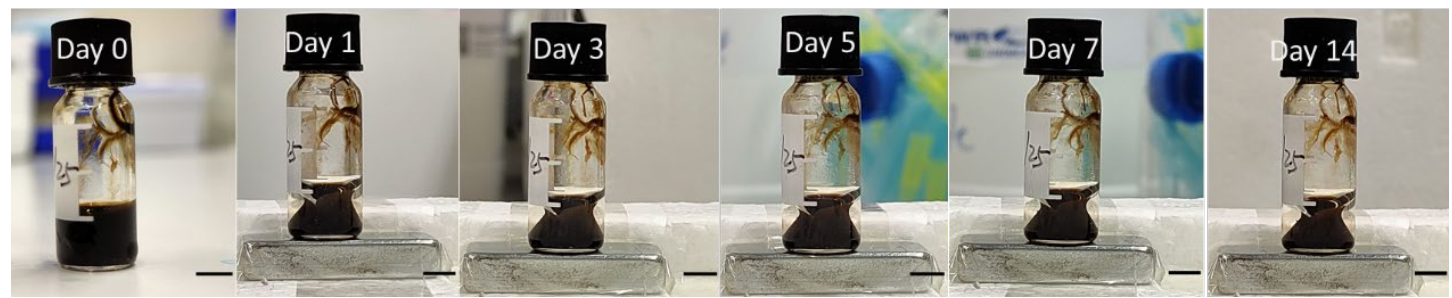

Figure S6. Photos of the ferrogels with different MIONR concentrations in the presence of the magnetic fields for fourteen days. Composites with $C_{\mathrm{PIC}}=2 \mathrm{mg} \mathrm{mL}^{-1}$ and $C_{\mathrm{MIONRs}}=6 \mathrm{mg} \mathrm{mL}^{-1}$ (A) and $25 \mathrm{mg} \mathrm{mL}^{-1}$ (B), scale bar $5 \mathrm{~mm}$. The images of $C_{\text {MIONRs }}=6 \mathrm{mg} \mathrm{mL}^{-1}$ showed that in none of the composites any magnetic field-induced contraction was observed, not even after 14 days. The composites with $C_{\mathrm{MIONRs}}=25 \mathrm{mg} \mathrm{mL}^{-1}$ showed some syneresis at day 1 as observed in the top of the images. The block neodymium magnet (Webcraft GmbH \#Q-30-15-06-Z) was used to provide the magnetic fields. The field strength at the surface of the magnet is around $260 \mathrm{mT}$, which was measured by a Gauss meter (HGM09s). Note that in none of the sample particle chain-like structuters or precipitation was observed, suggesting that the particles are captured in the network.

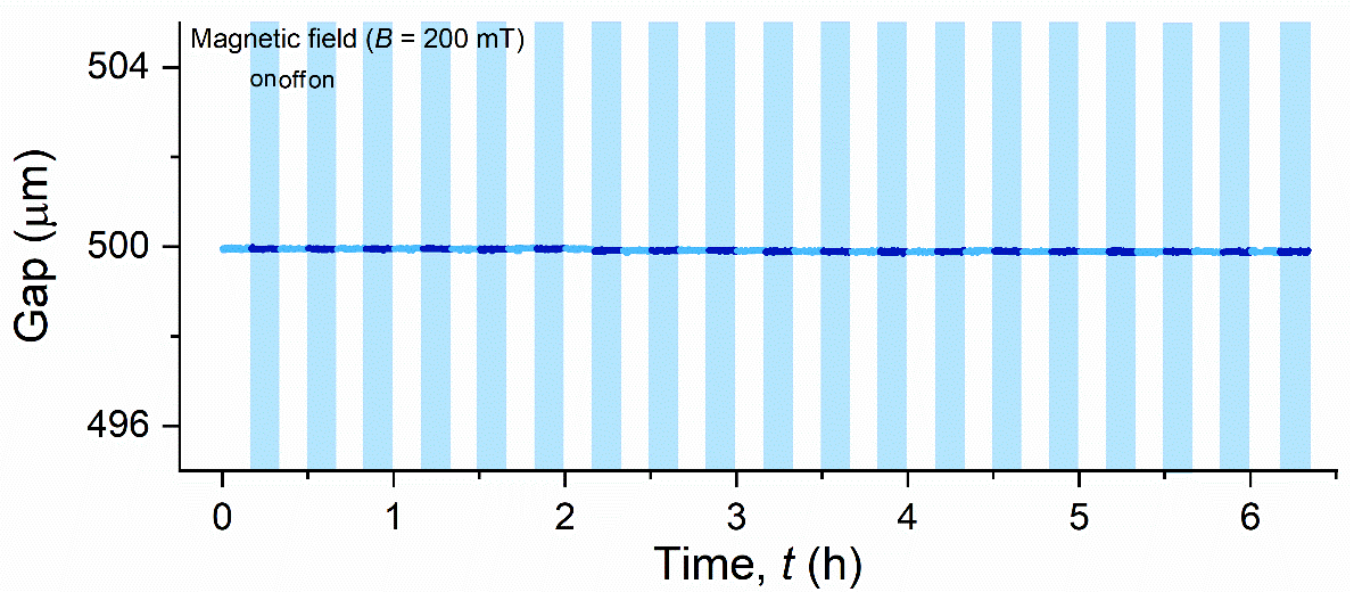

Figure S7. The gap size of the geometry in the absence or presence of an external magnetic field during the reversibility measurement by nineteen $20 \mathrm{~min}$ cycles ( $10 \mathrm{~min}$ with field on, and $10 \mathrm{~min}$ with field off). The rheometer will adjust the gap size when the normal force $F_{\mathrm{N}}$ on the $20 \mathrm{~mm}$ top plate exceeds $\left|F_{\mathrm{N}}\right|>0.5 \mathrm{~N}$. The results show that the gap is constant even in the presence of an external magnetic field, indicating the composites did not contract. Conditions: the concentrations of PIC and MIONRs are $0.5 \mathrm{mg} \mathrm{mL}^{-1}$ and $25 \mathrm{mg} \mathrm{mL}^{-1}$, respectively. 

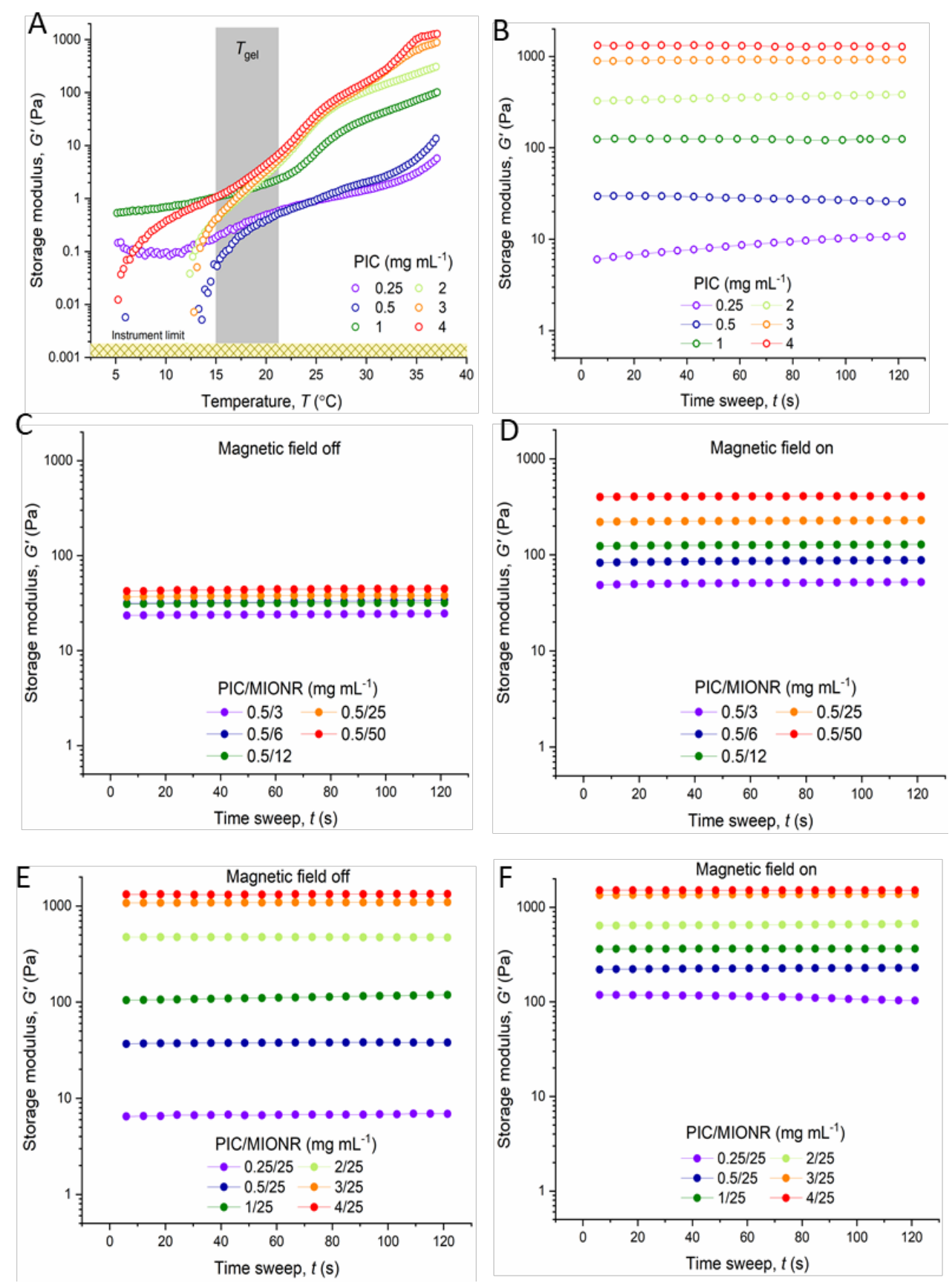

Figure S8. The mechanical properties of PIC gels and PIC-MIONR composites. (A,B) The storage modulus $G^{\prime}$ of different concentration PIC gels as a function of temperature (A) and in a following time sweep at $T=37^{\circ} \mathrm{C}(\mathrm{B})$. The gelation temperature is around $15 \sim 20{ }^{\circ} \mathrm{C}$. (C-F) Time sweeps of PIC-MIONR composite gels with different concentrations of MIONRs in the absence (C) and presence (D) of an external magnetic field $\boldsymbol{B}=1 \mathrm{~T}$ and with different PIC concentrations in the absence (E) and presence (F) of an external magnetic field $\boldsymbol{B}=1 \mathrm{~T}$. 

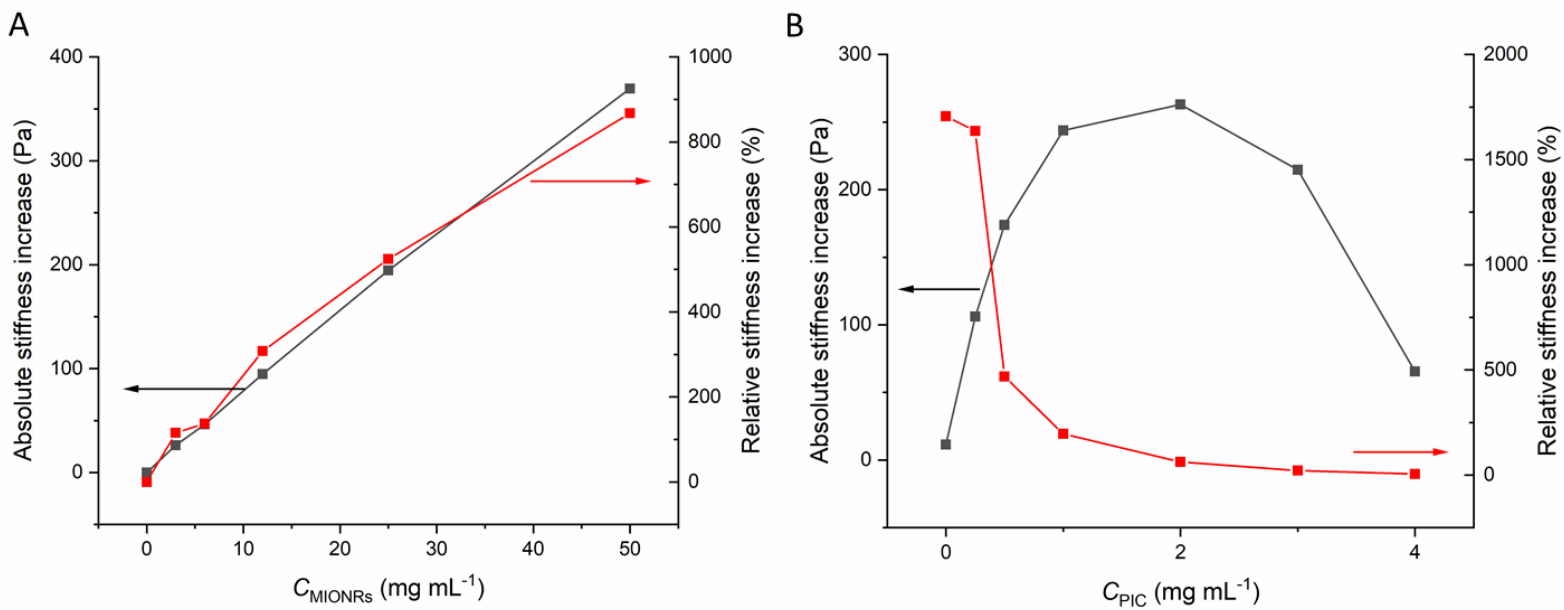

Figure S9. (A) Absolute (black data) and relative (red data) changes in stiffness between $B=0$ and $1 \mathrm{~T}$ as a function of MIONRs concentration in composite with $C_{\mathrm{PIC}}=0.5 \mathrm{mg} \mathrm{mL}^{-1}$; (B) Absolute (black data) and relative (red data) changes in stiffness between $B=0$ and $1 \mathrm{~T}$ against PIC concentration in composites with $C_{\mathrm{MIONR}}=25 \mathrm{mg} \mathrm{mL}^{-1}$.
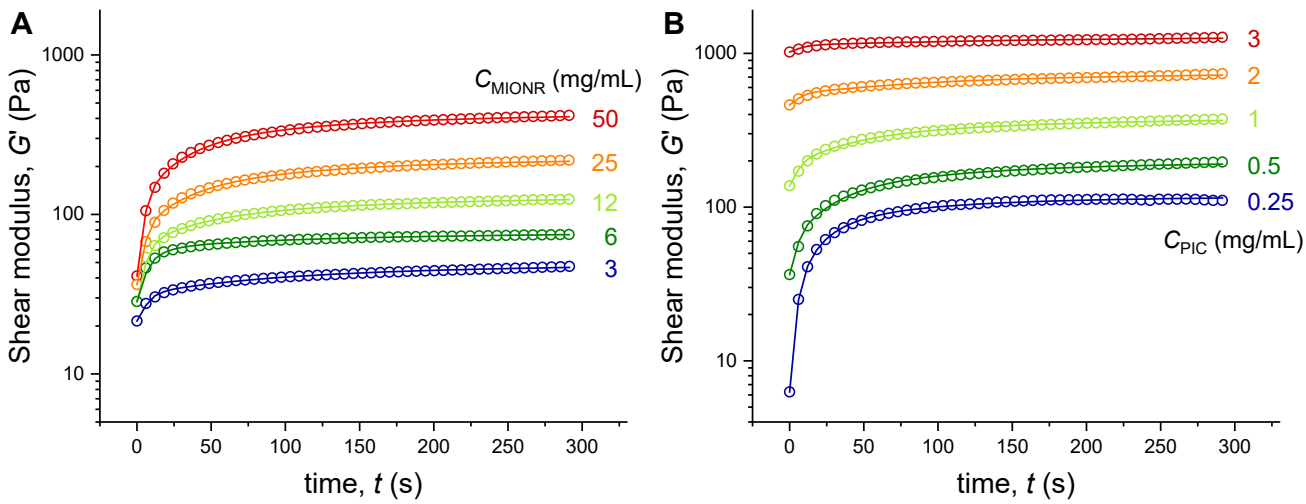

Figure S10. Evolution of (A) storage modulus $G^{\prime}$ with composites $\left(C_{\mathrm{PIC}}=0.5 \mathrm{mg} \mathrm{mL}^{-1}\right)$ with varying MIONR concentrations in time under an external magnetic field that is ramped up from $0 \mathrm{~T}$ to $1 \mathrm{~T}$ within 5 mins.; and (B) $G^{\prime}$ for composites $\left(C_{\mathrm{MIONR}}=25 \mathrm{mg} \mathrm{mL}^{-1}\right)$ with varying PIC concentrations in time under an external magnetic field that is ramped up from $0 \mathrm{~T}$ to $1 \mathrm{~T}$ within 5 mins. To study the dynamic kinetics, we ignored the data of $G^{\prime}$ at $C_{\mathrm{PIC}}=4 \mathrm{mg}$ $\mathrm{mL}^{-1}$ of PIC, because there is no magnetic stiffening effect in this condition (Fig.2c and $\mathrm{d}$ in the manuscript). 


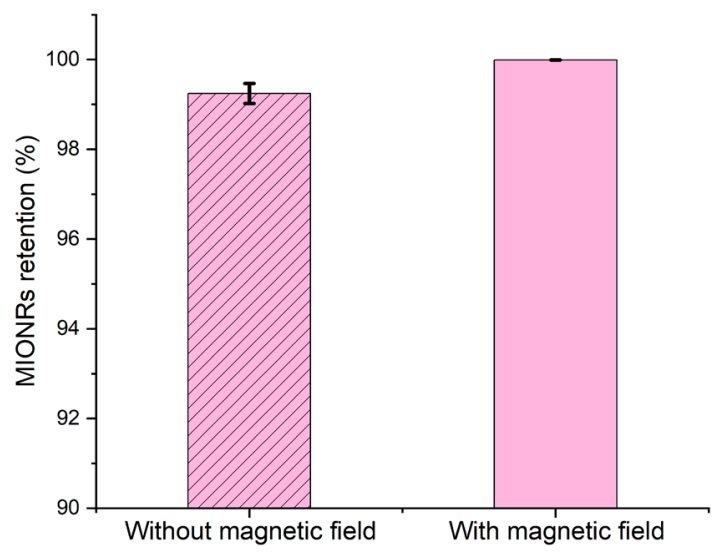

Figure S11. MIONRs-retention of PIC/Matrigel/MIONRs gel composites in the absence or presence of a magnetic field. The results reveal a very high retention capacity for the gels, irrespective of the presence of the magnetic field. Conditions: the concentrations of the PIC, Matrigel and MIONRs are $2 \mathrm{mg} \mathrm{mL}^{-1}, 3.52 \mathrm{mg} \mathrm{mL}^{-1}$ and $25 \mathrm{mg} \mathrm{mL}^{-1}$, respectively. The samples were kept at $37^{\circ} \mathrm{C}$ for 3 days.

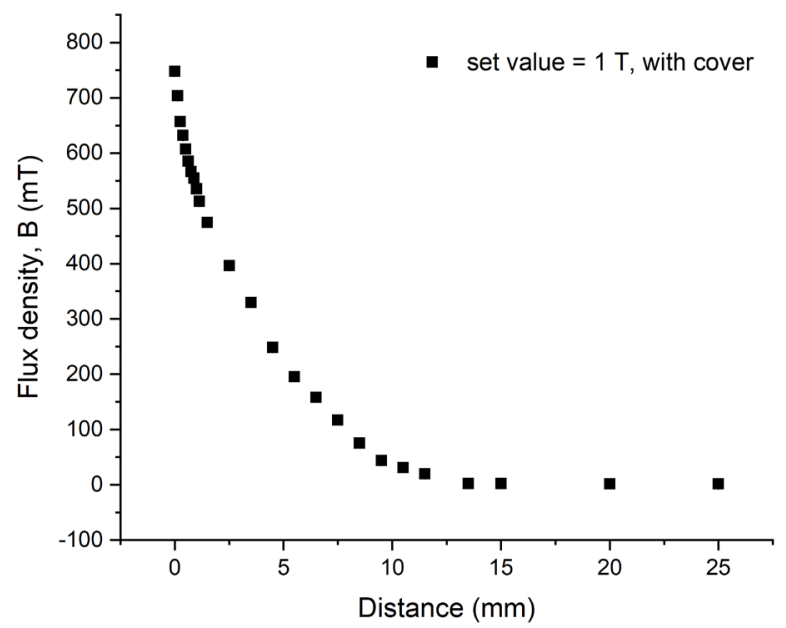

Figure S12. Measured magnetic field gradient of the magneto-rheometer (with the cover) as a function of distance from the top center of the magneto set-up as measured using a Gauss meter. 

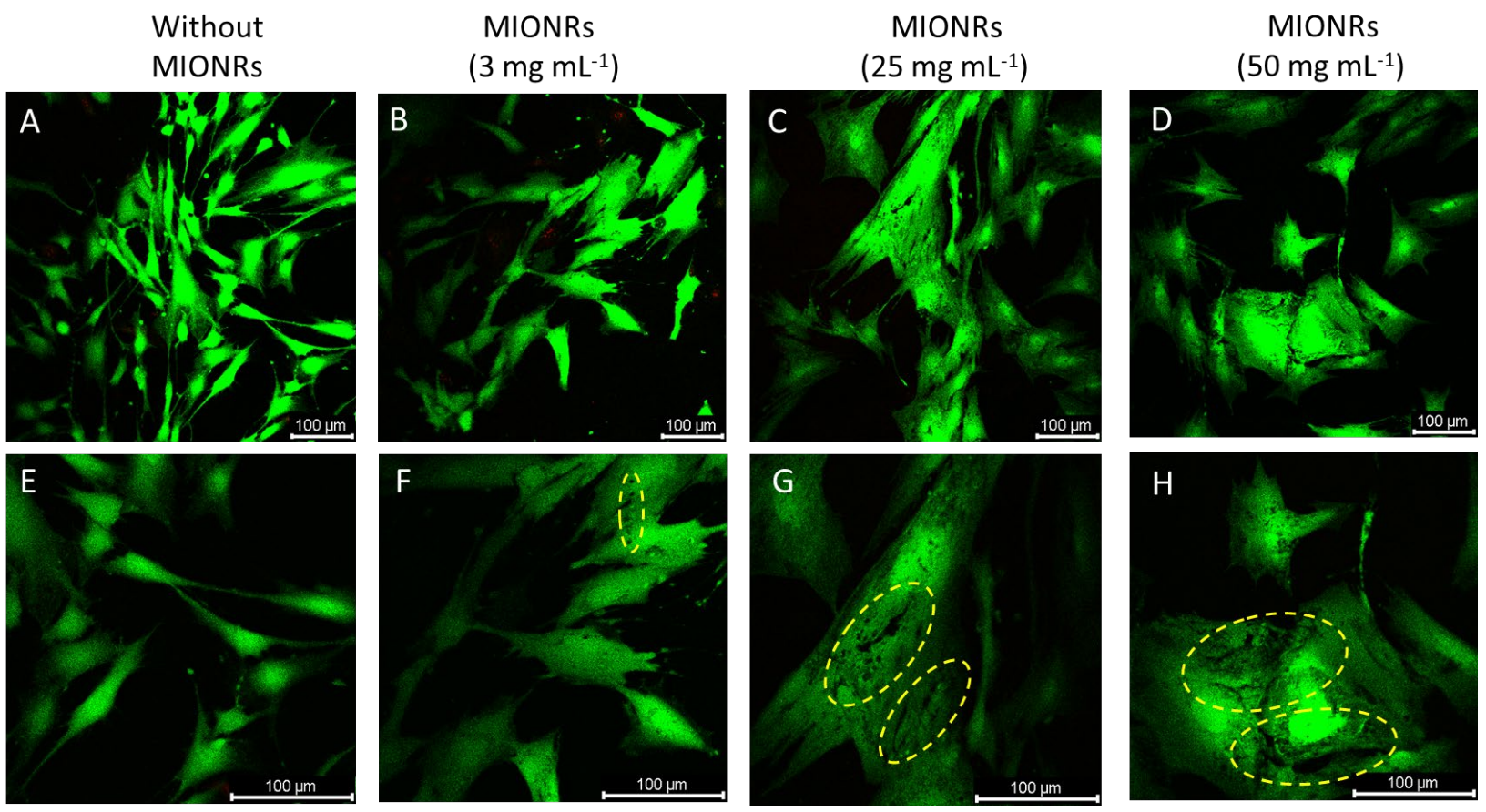

Figure S13. Fluorescence images of human foreskin fibroblasts after Live /Dead assay 72 after seeding in 2D culture with different concentrations of MIONRs in the absence of a magnetic field. For the live-dead staining, green stands for live cells, red stands for dead cells. E-H images are high resolution zooms corresponding to A-D, respectively. The yellow dash lines indicate the locations of MIONRs. Scale bars are $100 \mu \mathrm{m}$ for all panels.

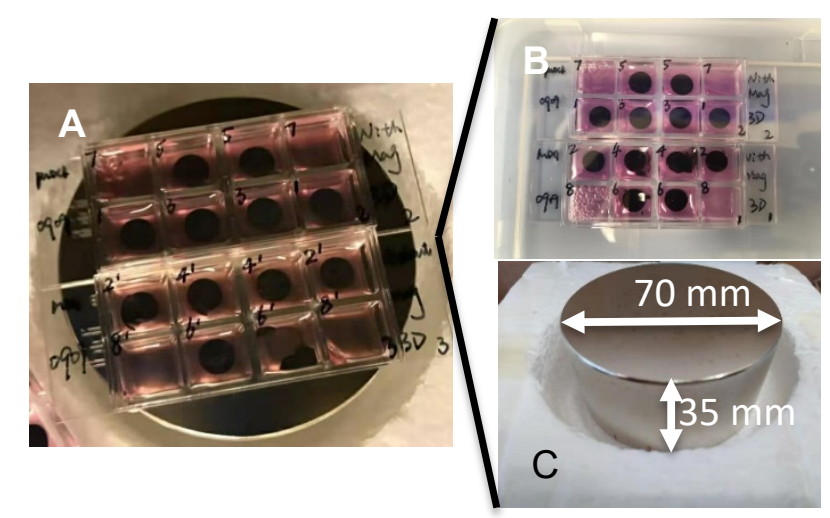

Figure S14. Photo A) of cell culture experiment in the presence of an external magnetic field. B) 8 well plates. C) A permanent neodymium magnet (Webcraft GmbH S-70-35-N). The diameter of the magnet is $70 \mathrm{~mm}$, and the height is $35 \mathrm{~mm}$. It is big enough to place two 8 well plates on it. 


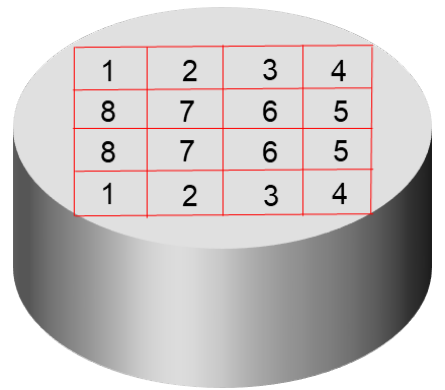

Figure S15. The scheme of the magnet used for cell culture experiment. The magnet is divided into two parts, where two 8-well plates can be placed on the magnet. The corresponding magnetic fields at each position are given in Table S4.
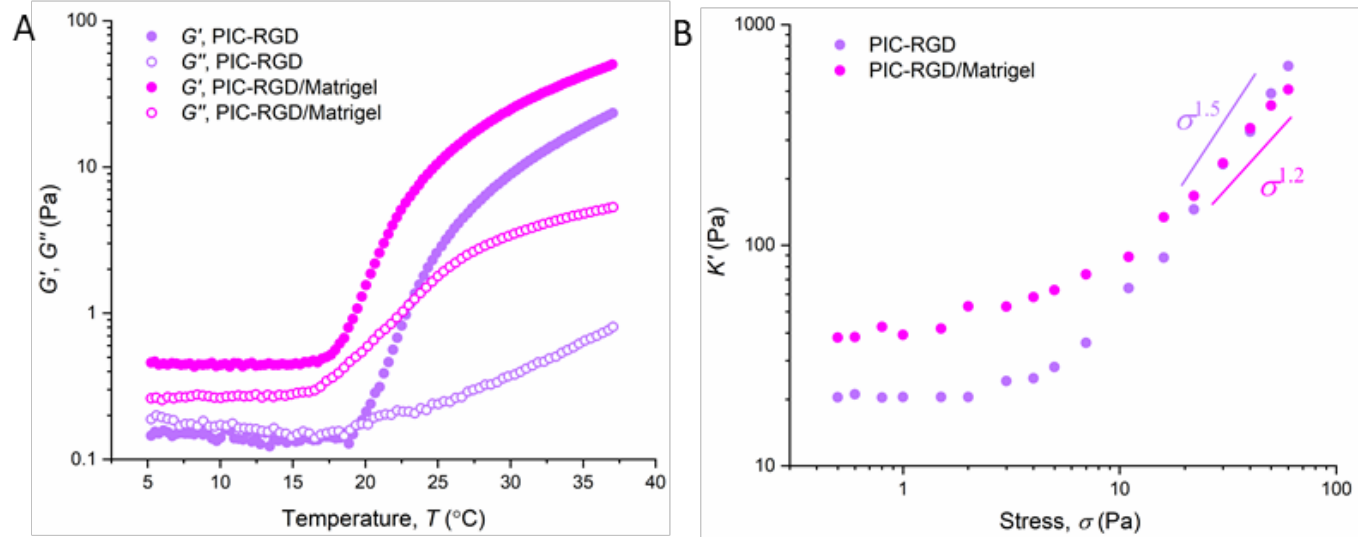

Figure S16. The mechanical properties of PIC-RGD and PIC-RGD/Matrigel mixtures. A) Temperature ramp of the storage modulus $G^{\prime}$ and loss modulus $G^{\prime \prime}$ for PIC-RGD gels and PIC-RGD/Matrigel hybrid. The stiffness increases from $25 \mathrm{~Pa}$ to $48 \mathrm{~Pa}$ after the introduction of Matrigel. B) Differential modulus $K^{\prime}$ as a function of stress. The stiffening index $m$ of PIC-RGD/Matrigel is 1.2, which is lower than that of PIC-RGD $(m=1.5)$. It means that the introduction of Matrigel into PIC-RGD network decreases the stiffening response of PIC, which plays an important role in the difference of these two gel systems. 

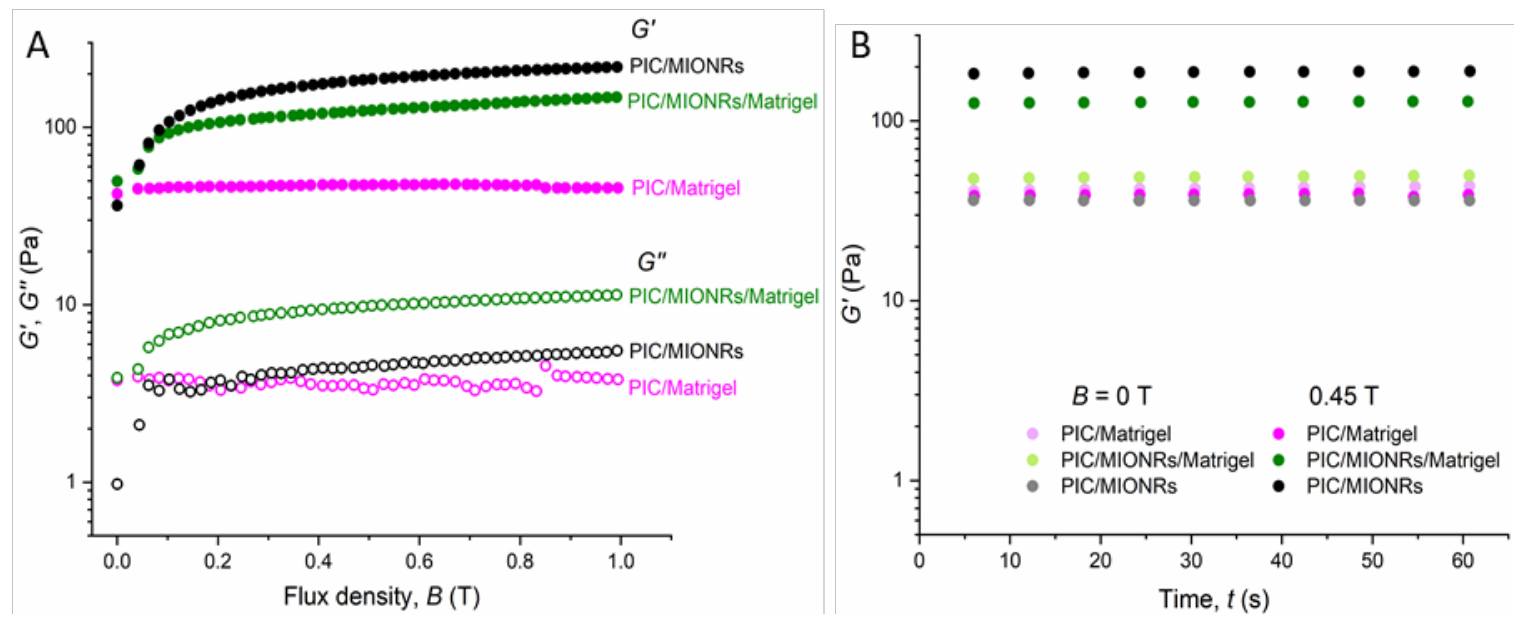

Figure S17. The stiffness of gels that were used for cell culture. A) The storage modulus $G^{\prime}$ and loss modulus $G^{\prime \prime}$ of PIC/Matrigel gels with and without MIONRs as a function of flux density. B) $G^{\prime}$ and $G^{\prime \prime}$ of the gels with and without MIONRs in the absence and presence of the external magnetic field at $450 \mathrm{mT}$ as a function of time to quantify the stiffness of gels when doing cell culture. Conditions: the concentrations of PIC-RGD and MIONRs are $2 \mathrm{mg} \mathrm{mL}^{-1}$ and $25 \mathrm{mg} \mathrm{mL}^{-1}$, respectively, the concentration of Matrigel is $3.52 \mathrm{mg} \mathrm{mL}^{-1}$. Note: The PIC used for this study was a different batch compared with the materials (used for doing mechanical properties experiments) in the rest of the manuscript. In addition, the cell-adhesive peptide GRGDS was conjugated to PIC. The introduction of the peptide reduces the stiffness of PIC. ${ }^{7}$

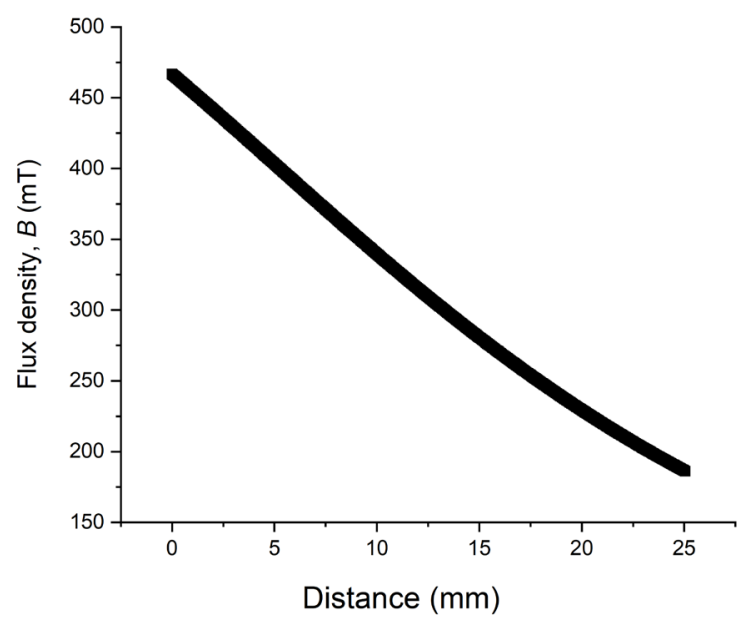

Figure S18. Measured magnetic field gradient of the neodymium magnet as a function of distance from the top center of the magnet. 
Table S1. The fitting results of the experimental stiffening data of PIC/MIONRs hydrogels from Figure $2 b, d$ and Figure 3a.

\begin{tabular}{ccccc}
\hline $\begin{array}{c}C_{\text {MIONR }} \\
\left(\mathrm{mg} \mathrm{mL}^{-1}\right)\end{array}$ & $\begin{array}{c}C_{\text {PIC }} \\
\left(\mathrm{mg} \mathrm{mL}^{-1}\right)\end{array}$ & $\begin{array}{c}C_{\text {MIONR }} \cdot C_{\text {PIC }} \\
\left(\mathrm{mg}^{2} \mathrm{~mL}^{-2}\right)\end{array}$ & $\begin{array}{c}G^{\prime}=K^{\prime} \\
(\mathrm{Pa})\end{array}$ & $\begin{array}{c}\sigma \\
(\mathrm{Pa})\end{array}$ \\
\hline 3 & 0.5 & 1.5 & 49 & 3 \\
6 & 0.5 & 3 & 80 & 4.4 \\
12 & 0.5 & 6 & 125 & 7 \\
25 & 0.5 & 12.5 & 231 & 12.1 \\
50 & 0.5 & 25 & 412 & 21 \\
\hline 25 & 0.25 & 6.25 & 112 & 6.8 \\
25 & 0.5 & 12.5 & 211 & 12.7 \\
25 & 1 & 25 & 368 & 22 \\
25 & 2 & 50 & 686 & 35 \\
25 & 3 & 75 & 1237 & 46 \\
25 & 4 & 100 & 1422 & 53 \\
\hline
\end{tabular}

Table S2. Fitting results of the experimental stiffening data (Figure S10A) to equation (1) for PIC-based hybrid hydrogels with different concentrations of MIONRs. Note that the fitting parameter $t_{1}$ was shared for all data sets during the fitting procedure. Conditions: $0.5 \mathrm{mg} \mathrm{mL}^{-1}$ of PIC with different concentrations of MIONRs.

\begin{tabular}{ccccccc}
\hline $\begin{array}{c}C_{\text {MIONR }} \\
\left(\mathrm{mg} \mathrm{mL}^{-1}\right)\end{array}$ & $G_{0 \mathrm{~T}}^{\prime}$ & $\Delta G_{\max }^{\prime}$ & $t_{1}$ & $t_{2}$ & $A_{1}$ & $A_{2}$ \\
$(\mathrm{~Pa})$ & $(\mathrm{Pa})$ & $(\mathrm{s})$ & $(\mathrm{s})$ & & \\
\hline 3 & 21 & 145 & 6.6 & 135 & 0.11 & 0.10 \\
6 & 28 & 136 & & 204 & 0.31 & 0.08 \\
12 & 28 & 284 & & 91 & 0.22 & 0.16 \\
25 & 36 & 429 & & 71 & 0.22 & 0.25 \\
50 & 41 & 698 & & 64 & 0.27 & 0.33 \\
\hline
\end{tabular}

Table S3. Fitting results of the experimental stiffening data (Figure S10B) to equation (1) for PIC-based hybrid hydrogels with different concentrations of PIC. Note that the fitting parameter $t_{1}$ was shared for all data sets during the fitting procedure. Conditions: $25 \mathrm{mg} \mathrm{mL}^{-1}$ of MIONRs with different concentrations of PIC.

\begin{tabular}{ccccccc}
\hline $\begin{array}{c}C_{\mathrm{PIC}} \\
\left(\mathrm{mg} \mathrm{mL}^{-1}\right)\end{array}$ & $\begin{array}{c}G_{0 \mathrm{~T}}^{\prime} \\
(\mathrm{Pa})\end{array}$ & $\begin{array}{c}\Delta G_{\max }^{\prime} \\
(\mathrm{Pa})\end{array}$ & $\begin{array}{c}t_{1} \\
(\mathrm{~s})\end{array}$ & $\begin{array}{c}t_{2} \\
(\mathrm{~s})\end{array}$ & $A_{1}$ & $A_{2}$ \\
\hline 0.25 & 6 & 327 & 6.6 & 38 & 0.17 & 0.19 \\
0.50 & 36 & 479 & & 59 & 0.14 & 0.22 \\
1.0 & 137 & 1653 & & 73 & 0.07 & 0.09 \\
2.0 & 462 & 3302 & & 123 & 0.04 & 0.06 \\
3.0 & 1019 & 3446 & & 194 & 0.05 & 0.04 \\
\hline
\end{tabular}


Table S4. The field strength of the magnet at different positions. Error is standard deviation over 3 measurements.

\begin{tabular}{lllll}
\cline { 1 - 2 } \cline { 5 - 6 } \cline { 5 - 6 } Position & $\boldsymbol{B}(\mathrm{mT})$ & & Position & $\boldsymbol{B}(\mathrm{mT})$ \\
\hline 1 & $500.1 \pm 2.2$ & & 5 & $464.2 \pm 3.8$ \\
2 & $479.5 \pm 2.5$ & & 6 & $444.2 \pm 4.1$ \\
3 & $480.7 \pm 1.9$ & & 7 & $440.1 \pm 2.7$ \\
4 & $494.3 \pm 4.3$ & & 8 & $469.5 \pm 5.2$ \\
\hline
\end{tabular}

\section{Additional references}

(1) Lou, L.; Yu, K.; Zhang, Z.; Huang, R.; Zhu, J.; Wang, Y.; Zhu, Z., Dual-mode protein detection based on Fe $3 \mathrm{O}$ 4-Au hybrid nanoparticles. Nano Res. 2012, 5, 272-282.

(2) Hong, Y.; Shi, H.; Shu, X.; Zheng, Y.; Zhang, Y.; Wu, Y., Controlled synthesis of hollow magnetic Fe3O4 nanospheres: Effect of the cooling rate. Particuology 2017, 33, 24-28.

(3) Xu, W.; Wang, M.; Li, Z.; Wang, X.; Wang, Y.; Xing, M.; Yin, Y., Chemical transformation of colloidal nanostructures with morphological preservation by surface-protection with capping ligands. Nano Lett. 2017, 17, 2713-2718.

(4) Kouwer, P. H. J.; Koepf, M.; Le Sage, V. A. A.; Jaspers, M.; van Buul, A. M.; Eksteen-Akeroyd, Z. H.; Woltinge, T.; Schwartz, E.; Kitto, H. J.; Hoogenboom, R.; Picken, S. J.; Nolte, R. J. M.; Mendes, E.; Rowan, A. E., Responsive biomimetic networks from polyisocyanopeptide hydrogels. Nature 2013, 493, 651-655.

(5) Schoenmakers, D. C.; Rowan, A. E.; Kouwer, P. H. J., Crosslinking of fibrous hydrogels. Nat. Commun. 2018, 9, 2172.

(6) Das, R. K.; Gocheva, V.; Hammink, R.; Zouani, O. F.; Rowan, A. E., Stress-stiffening-mediated stem-cell commitment switch in soft responsive hydrogels. Nat. Mater. 2016, 15, 318-325.

(7) Liu, K.; Mihaila, S. M.; Rowan, A.; Oosterwijk, E.; Kouwer, P. H. J., Synthetic Extracellular Matrices with Nonlinear Elasticity Regulate Cellular Organization. Biomacromolecules 2019, 20, 826-834.

(8) Jaspers, M.; Vaessen, S. L.; van Schayik, P.; Voerman, D.; Rowan, A. E.; Kouwer, P. H. J., Nonlinear mechanics of hybrid polymer networks that mimic the complex mechanical environment of cells. Nat. Commun. 2017, 8, 15478.

(9) An, H.; Picken, S. J.; Mendes, E., Enhanced hardening of soft self-assembled copolymer gels under homogeneous magnetic fields. Soft Matter 2010, 6, 4497-4503.

(10) de Almeida, P.; Jaspers, M.; Vaessen, S.; Tagit, O.; Portale, G.; Rowan, A. E.; Kouwer, P. H. J., Cytoskeletal stiffening in synthetic hydrogels. Nat. Commun. 2019, 10, 1-8.

(11) Koenderink, G. H.; Dogic, Z.; Nakamura, F.; Bendix, P. M.; MacKintosh, F. C.; Hartwig, J. H.; Stossel, T. P.; Weitz, D. A., An active biopolymer network controlled by molecular motors. Proc. Nat. Acad. Sci. U. S. A. 2009, 106, 15192-15197.

(12) Jiles, D., Introduction to magnetism and magnetic materials. CRC press: 2015. 